\title{
The Rejuvenation of Equivalence Paradigm in Literary Texts: Equimediation as the Seul et Alleiniges Paradigm in Translation
}

\author{
Alireza Akbari \\ Faculty of Foreign Languages, University of Isfahan, Iran \\ Email: dictogloss@gmail.com \\ Mohammadtaghi Shahnazari \\ Faculty of Foreign Languages, University of Isfahan, Iran \\ Email:m_tshah@yahoo.com
}

Doi:10.5901/mjss.2015.v6n4s2p438

\section{Abstract}

\begin{abstract}
Currently, conferring a durable and fix definition of "Equivalence" is a drudgery task and activity in Translation Studies (TS). That is owing to the fact that the former demarcations substantiate as unworkable, unexploitable, and inefficient in this area. Equivalence in TS is a cardinal facet which needs to be paid much more heed. To ratify the essence of equivalence in translation, some paradigms vulgarized for their functionality. Paradigms such as Equivalence, Purpose, and Description are the core ones in the intended field. On the flipside, their fame never continue to exist so as to verify the very nature of equivalence. This is due to the fact that these paradigms did not work well on the whole facets of equivalence. The present study seeks to investigate on one fresh paradigm in equivalence known as Equimediation Paradigm (EP). This paradigm is made of five major phases as Strong Equivalents (SE), Weak Equivalents (WE), Brittle Equivalents (BE), Degree of Intensity $(D I)$, and Degree of Occurrence (DO) to show its feasibility and speakability of the concealed rationale behind the literary text. In the circle of translation, translator is treated as a robust mediator in order to interlink the source rationality to the target one. Equimediation paradigm spies out the void and $x$-areas in TS to substantiate the vital particularities of equivalence in diverse fields. As time elapses, Equimediation Paradigm seeks to peruse Source-Target Reconciliation and Deep-Surface Layer of language so that the translator can meet the needs of the source-target reader as part of the team in this paradigm.
\end{abstract}

Keywords: Equivalence, Equimediation Paradigm, Source-Target Reconciliation, Deep-Surface Layer of language

\section{Introduction}

Nowadays, New Translation Studies concentrates more on cross-cultural or inter-cultural communications. It is completely natural to say that communication requires establishing one stable paradigm so as to transmit the approximate function, form, and meaning of the source language into the target one. In this process, Equivalence Paradigm is notably worth noticing. Establishing and functionalizing Equivalence Paradigm in translation is an ordeal task despite its feasibility and practicality. As Catford (1965) points out that:

"The central problem of translation practice is that of finding target language equivalents. A central task of translation theory is that of defining the nature and condition of the translation equivalence (p.21)."

Generally speaking, replacing the textual material of the source into the textual material of the target one requires familiarizing with the two poles (Source Language and Target Language) in translation. However, in recent decades; most of the translators or interpreters distort and neglect the validity of equivalence in their translations just so as to convey the essence of the text.

Prior to the act of translating, translator should consider two aspects of equivalence namely: (1) Equivalence is definitional and (2) Equivalence is feasible. The former alludes to translator's options to opt for the near and proximate equivalents to ameliorate the process of translation. And the latter refers to the effects on readers. It means that choosing suitable and appropriate equivalent causes the text to convince the reader which is the ultimate aim of equivalence paradigm in Translation Studies. However, most of the translators cover the first trait of equivalence due to the fact that they are unable to schedule their time to opt for the right and convincing equivalent. In this case, mutually agreement amongst the source language, the translator as the mediator, target language, and target readers will be violated and 
labeled the translation as an artificial or business rendering. Therefore, attempts should be made to connect the two facets of equivalents simultaneously.

In the second half of twentieth century, theorists of translation faced with choosing the right and proper equivalents for their renderings. One of the most and robust frontiers against equivalence paradigm was Structural Linguistics. The real and fix notion of structuralism comes from the viewpoint of Wilhelm Von Humboldt, Edward Sapir and Benjamin Whorf observing diverse worlds along with different languages. Mostly, they scrutinize language as some sets of relation and they do not pay attention to the meaning, form, and function of language. According to structuralism's notion, the text by nature is untranslatable and no one cannot discover the same value or function in the target language. Nevertheless, in the long run; Equivalence Paradigm gave rise to its climax in that it inspects the equal values in source and target languages. Therefore, most of the translation turned to be artificial since the reader could not persuade him or herself as the persuasive translation. As the time goes by; equivalence paradigm progressed and ameliorated since it could answer some of the translators' queries on natural equivalence. Then Equivalence Paradigm came to the scene as Natural Equivalence.

Nida and Taber defined Natural Equivalence as "reproducing in the receptor language the closest natural equivalent of the source language message $(1969$, p.12)." In this direction, translation equivalence would be regarded as one-to-one correspondence. It means that one element of the source is translated by one corresponding element in the target language. Andrew Chesterman (1996/2005) argues that natural equivalence will be treated as convergent similarities in which it conveys same qualities of the source text and later he depicts natural equivalence as $A \leftrightarrow B$. This interaction is completely reversible as source and target language will be treated the same. But, the problem is that how can natural or convergent equivalents verify new terminologies/information in translation? Or how can convergent similarities corroborate the psychological nature of the source language text?

Due to unworkability of the natural equivalence, most of the translators propose the directional equivalence in their translations. Directional equivalence can be defined as "a source language text to a target language text which is as close an equivalent as possible and presupposes an understanding of the content and style of the original (Wills, 1982, p.32)."

Noteworthy, directional equivalence behaves as one-to-two correspondence as one textual material in the source language can be utilized by two or more textual materials in the target language. In this respect, Andrew Chesterman (1996/2005) addressed this kind of equivalence as "divergent similarities" since its relation is asymmetric along with various roles and expectations. He represents divergent similarities as $A \rightarrow B, C$, and $D$... Divergent similarities positions itself in irreversible way in which the translator cannot render the target language text like that of the source at the same time.

Directional equivalence suffers from some shortcomings such as source-target asymmetries and superiority of the source text. Equivalence paradigm would be investigated on the other paradigms such as Purpose paradigm (Skopos), Description Paradigm, and Localization Paradigm which will be expatiated thoroughly in literature review of this article.

Finally, there are some challenges on Equivalence Paradigm which puts this paradigm in the lowest position amongst others. This present study seeks to investigate on unworkabilities of translational paradigms in New Translation Studies and then proposes one new paradigm known as "Equimediation Paradigm" to show void and ambiguous areas in translation. Equimediation paradigm inspects the higher and lowest positions (outcomes) of the words in order to saturate the needs of the reader. In this process, Equimediation paradigm considers all possibilities in translation such as comprehensibility, translatability, and untranslatability. It is tried to depict the well-balanced scheme and explain the nature and subcategories of the intended paradigm in Translation Studies.

\section{Review of Literature}

\subsection{Equivalence Paradigm}

Equivalence is a term in translation which broadly covers information outside the field of enquiry. Therefore, it is better to depict and explain the general definition of this term so as to get acquainted with the nature of equivalence. According to House (1997):

"The notion of equivalence is the conceptual basis of translation and, to quote Catford (1965), 'the central problem of translation practice is that of finding the TL (Target Language) equivalents. A central task of translation theory is therefore that of defining the nature of conditions of translation equivalence' (p. 21)" (p.25)."

In this sentence, one has to discover the suitable and appropriate element in the target language having the same 
potentials as the source language elements in this regard. Therefore, replacing textual material of the source text into the target language textual material is of great importance in equivalence paradigm. Equivalents in translation cause the renderings more to be operationalized as they inspects the superficial aspects of the equivalents. Operationalizing the translation needs to schedule equivalence via Machine Translation since it scrutinizes the possible range of equivalents in the target language.

In this connection, Jäger (1989) explains that:

\begin{abstract}
"Auf dem Hintergrund der modernen übersetzungstheoretischen Konzeptionen, die daruaf abzilen, den Text in der Sprachmittlung ganzheitlich zu erfassen, entsteht zwangsläufig die Frage, ob die auf die Aufdeckung und Beschreibung von Äquivalenz betziehungen gerichtenen Untersuchungen überhaupt einen Sinn haben. Wir würden diese Frage ubedingt positiv beantworten und haben dabei einen besonders ansprochsvollen Bewährungsfall der übersetzungswissenschaft vor Augen, das automatische Übersetzen. (p.33)."
\end{abstract}

From the mentioned definition, one can conclude that the ultimate aim of equivalence paradigm is to deal with Machine Translation covering the surface structure of the source and target language text. However, the problem is that, equivalence paradigm will not be able to saturate the needs of the reader via machine translation. According to Akbari (2014a, p. 5-8) Machine Translation cannot cover the whole framework of equivalence such as (1) Syntactic Problem, (2) Natural Language Inter-translatability, (3) Idioms, Slangs, and Expressions, (4) Lexical Ambiguity, (5) Paralinguistic Problems, and (6) Stylistic Effects of Translation. Therefore, equivalence paradigm in Machine Translation is just utilized for superficial renderings of the text to convey near concepts of the source language into the target one.

It is necessary to explain that equivalence in Equivalence Paradigm is relational since no two equivalents can cover the same topics in the target language. Every equivalent has its own potential to be used in the target language to transfer the meaning. But, the important fact is that language patterning and language sensitivity are of great important to shape the real nature of equivalents in translation (Akbari, 2014b, p).

In this connection, Nida (1986) expressed that:

"There are no two stones alike, no flowers the same, and no two people who are identical. Although the structure of DNA in the nucleus of their cells may be the same, such persons nevertheless differ as the result of certain developmental factors. No two sounds are ever exactly alike, and even the same person pronouncing the same words will never utter it in an absolutely identical manner (p.60)."

Everything in this world is in state of suspense, and one cannot expound it by ins and outs. This is completely true of equivalence in Translation Studies. But, the important issue here is what Nida says "Although the structure of DNA in the nucleus of their cells may be the same". Every word emanates from the same source of comprehension and utilization. In spite the fact that no two equivalents are the same, the translator can approximate to the deep layer of the words in order to decipher the point of similarities. Therefore, establishing the well-defined procedures in equivalence paradigm to find the same equivalents is an arduous task yet possible.

\title{
2.2 Purpose Paradigm
}

Equivalence in this paradigm will be treated differently in compared with Equivalence Paradigm. In this category, equivalence will be treated via its purpose, message, and data-driven based. One of the first precursor of this paradigm dates back to Hans Vermeer (1978) who proposed this paradigm to corroborate the nature of target language text. He sees translation and equivalence in a circle of function in this direction. Apart from Hans Vermeer, the founder of Skopos theory, Christian Nord (1988), Margaret Amman (1989), Hans Hönig and Paul Kussmaul (1982), and Holz Mänttäri (1984) are other scholars who work in this area to corroborate the nature of function and purpose in translation.

In purpose paradigm, some elements should be mentioned as the basis. First, the translator should get acquainted to the term of Purpose in translation. According to Skopos theory, purpose will be defined as aim, goal, or intended function of the text. It means that the translator should consider the text type or intended function of the source text prior to the act of translating. Every text has its own function to be transferred. Therefore, equivalence should be treated functionally in order to persuade the reader to get the gist of the source text. But, some scholars in this paradigm are in state of disagreement which puts this category/paradigm into jeopardy. Some treat equivalence on the source based, whereas others treat it as the target-sided notion. The second element to be taken into account is the role of the client. Client plays the most important role in this paradigm in which he/she gives some instructions to the translator about the way of translating. Saturating the needs of the reader is of high performable. Client in the target text decides on 
functionality of translation. Naturally, equivalence in this paradigm will be influenced by the clear instruction of the client. Therefore, text types, target-sidedness, the role of the client, and the clear instruction or translation brief are the key components in this paradigm. So, the translator acts like an expert who seeks to establish cross-cultural communication.

Purpose paradigm determines equivalents in a professional situation as the translator should get familiar to all rules of the target language texts in order to do well in this paradigm. Professional situation refers to the role of the client, translation brief or instruction, commission, and translation target-sidedness discussed before. Therefore, unlike the equivalence paradigm which considers just the two poles in translation (source and target languages sides), purpose paradigm regards many factors in equivalence process which covers all aspects of translation such as translator's liberation, empowerment, or even repression of translator's individuality (Amman, 1994). The other note is that purpose paradigm addresses ethical rule in target translation as it respects to the needs of the target reader. In this connection, Christiane Nord (1997, p123) introduces the term of "loyalty" which the translator has ethical obligation in two parties: (1) to the texts and (2) to the people. Nord (1997) then defines the term of loyalty as:

"This responsibility translators have toward their patterns in translational interaction. Loyalty commits the translator bilaterally to the source and the target sides. It must not be mixed up with fidelity or faithfulness, concepts that usually refer to the relationship holding between the source and the target texts. Loyalty is an interpersonal category referring to social relationship between people. (p. 125)."

In this connection, whether the translator sees the unworkability or impracticality of the equivalence into the target language, he or she can refuse to produce the translation. Therefore, it must be concluded that purpose paradigm concentrates fully on the role of client and the translator [expert] and it gives the right of rejection of translation to the translator. Equivalence in this paradigm covers superficial aspects of translation which meets the needs of the reader to get the overall gist of translation and equivalence will be treated just on target side aspects. Considering the role of the target language equivalents is the ultimate aim of this paradigm in this regard.

\title{
2.3 Description Paradigm
}

Description as its name suggests, it aims is describing rather than prescribing. Description Translation Studies (DTS) is the new perspective on translation during the 1970s by Gideon Toury (1995) investigated solutions of the Translation Studies. Toury (1985) expressed his rationality of DTS as:

\begin{abstract}
"Semiotically speaking, it will be clear that it is the target or recipient culture, or a certain section of it, which serves as the initiator of the decision to translate and of the translating process. Translating as the teleological activity par excellence is to large extent conditioned by the goals it is designed to serve, and these goals are set in, and by, the prospective receptor system. Consequently, translators operate first and foremost in the interest of the culture into which they are translating, and not in the interest of the source text, let alone the source culture (p. 18-19)."
\end{abstract}

Generally speaking, Toury puts his effort in the target language text and he more fully concentrates on the solution rather than the problem itself. Target-sidedness like purpose paradigm is the sole trait of description paradigm as it scrutinizes the function, product, and the process of the translation and translator.

Toury (1995) determines three levels of analysis for translation to approximate DTS more on the target level: (1) all that translation.......... can involve, (2) what it does involve under various set of circumstances, and (3) what it is likely to involve. This is quite clear that Toury pays more attention to the function of the translation since it involves some special conditions to be taken as granted. Therefore, Delabastita (2008, p. 234) explains more about Toury's three level of analysis as follows:

(1) Level of System: theoretical possibilities (can be)

For each translation problem or source text, it is possible to envisage a whole range possible or theoretical solution or target texts.

(2) Level of norms: culture bound constraints (should be)

On the intermediate level of norms, some of these possible relationships will be recommended or even required as being the only ones that can generate genuine translations, whereas others will be dismissed or even simply ignored.

(3) Level of performance: empirical discursive practice (is)

We can then observe which relationships have actually materialized in a given cultural settings. By definition, these empirical relationships constitute the subset of the possible relationships; their degree of frequency in a given cultural situation is the crucial indication that certain norms have been at work. 


\section{Discussion}

As it was explained previously, translation paradigms treat differently to the equivalence. None of the intended paradigms utilize equivalence in-between the source and target language. Localization paradigm treats cross-culturally but the product of this interaction results in a super-artificial equivalence in this direction. Any appropriate equivalence should consider the deep and surface structure of both source and target languages. The important note is how the renderer can utilize equivalents in source-target amalgamation? And will it be possible to do so? To what degree the translator can forward in making an acceptable equivalent in the target language. Saturating the needs of the reader will be the ultimate goal of equivalence paradigm in New Translation Studies. New Translation Studies concentrate more fully on the void and unclear spots in translation. New Translation Studies emanated from the impossibilities in translation.

In New Translation Studies, the translator encounter two way of thinking: (1) Source-Target Amalgamation and (2) Source-Target Dispersion. The former refers to the mixing and conglomerating the source and target languages such as culture, way of thinking, and way of translation utilized. And the latter alludes to discrepancies between the source and the target language contexts. The latter step is utilized from the inception of translation era. But, the former stage is the new scope in translation as it seeks to find out the possibilities and opportunities in translation. Translation will be understood easily by means of its homogeneity. It is the time to pass the red lines in translation studies, it is the time to decipher the impossibilities and make them possible in translation. And finally, it is the time to be in between the big poles in translation: the source and target languages contexts.

This study seeks to present a new paradigm of equivalence in translation "Equimediation Paradigm". Equimediation is the blending term composed of equivalence and intermediation. As it is clear, the translation will be treated between the equivalence paradigms (e.g. purpose, description paradigms) and intermediary step in translation. Prior to the act of translating, the translator should put him/herself in the gray spot. The final aim of the translator is to conglomerate the deep-surface and source-target with each other to saturate the needs of either source or target readers. In every field of study, there are some gray spots which are neglected by the researcher. These spots contain some pivotal and charted information which are able to direct the outcome of the research. In Translation Studies, these spots have been forgotten and yet they have not been used to clarify and divulge the extraordinary relations and factors. This study seeks to reveal one of the many shaded spots in translation in order to direct the translator to opt for the best equivalents in the target language context.

To help understanding the essence of study, the following terms should be expatiated.

(1) Weak Equivalents: any equivalents which are subject to forgetting and are in low level of usage. These kinds of equivalents are exposed to be cliché and temporary utilized in either source or target language such as cliché metaphors, slangs, expressions, allusions, simile, and temporal conversational slangs. For instance, avoid like the plague, acid test, alive and kicking, and Achilles heel.

(2) Strong Equivalents: these sorts of equivalents are not exposed to change, that is due to the fact that the backgrounds of the intended equivalents are extremely valuable in nature. Cultural terms such as adages, proverbs, moral maxims, fixed expressions, frozen expressions, and collocations. For examples, no pain no gain, "Always do what is right", the mule has more horse sense than a horse, and you can push people just so far.

(3) Brittle Equivalents: these types of equivalents are the most sensitive equivalents in the target language. Sensitive in cases which are completely pertained to the belief, creeds, and personality of people. This category should be taken cautiously in New Translation Studies. The important fact is that brittle equivalence is sometimes inclines to the source and sometimes to the target language context. This is possible when the target and source readers are completely aware of their language patterning and try to find some ways to connect them altogether. Euphemism (sweet talking), dysphemism (speaking offensively), and orthophemism (straight talking), and derogatories are in this category. For instance, 100, poo, Christ, Jesus, Lord, cur, and dopy.

(4) Degree of Intensity: degree of intensity refers to the power of the equivalents in the target language. Power of equivalents or equivalence potential is the particular situation either in the source or the target language which the reader hierarchize the power of the words. One of the most important factor in this item will be Wafting (e.g. Total Wafting, Partial Wafting, and Nativized Wafting) (Akbari, 2013) which scrutinizes the function of deep and surface structure at the same time. For instance, alpenglow, degauss, glitz, angst, Anschluss, blitz, Anglicism, and Seifenoper.

(5) Degree of Occurrence: degree of occurrence refers to the repetition of the equivalents in the context. It is labeled as degree of frequency. This factor is pertained to the number of words or equivalents which the 
translator utilizes in the target language context. Weak equivalents will be turned into low degree of occurrence and strong equivalents will be treated as high degree of occurrence in this essence.

To clarify the function of Equimediation paradigm in translation, it is better to reveal one important scheme so that a translator can be convinced to understand the fundamental aspects of this paradigm.

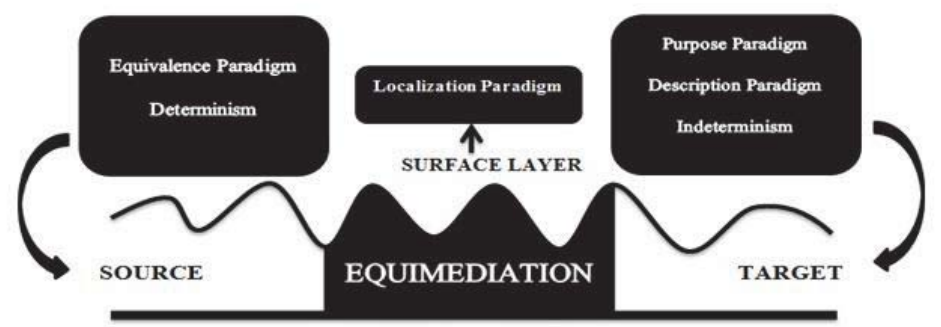

DEEP LAYER

Figure 1: Equimediation Paradigm

As explained before, Equimediation is the kind of paradigm which inspects equivalents into two rudimental factors: (1) Source-Target Amalgamation and (2) Deep-Surface Layer. These items play key roles in New Translation Studies aiming at clarifying the deep and surface layer of the translation either in the source or target language contexts. It should be noted that strong equivalents incline more to the deep layer source-target amalgamation and the weak one more on surface layer source-target amalgamation. Brittle equivalents sometimes incline to be strong enough and sometimes to be weak equivalents in this direction. Brittle equivalents are contextual-temporal-based in which they are used in different contexts along with various purposes.

One of the important factors in Equimediation paradigm is to determine the decision maker on the product of the translation. As expatiated early in the article, some said that the role of the client was of great importance and the process and product of the translation should be in accordance with the client's instructions. One the other hand some argued that role of a translator is the foremost part in rendering process and the translation should be twirled in the axle of the translator. The translator in this group may call an expert who knows ins and outs of the context in the target language. But, Equimediation Paradigm utilizes source, target, reader or client, translator, and the translation at the same time so as to persuade the reader on feasibility, applicability, and appropriateness of the translation. Needless to say, Equimediation paradigm is more coincided to the 'job specification' of Daniel Gouadec (2007) who explained client and the translator. Every person has the right to judge the translation. Therefore, the translator and the client should come in touch with each other. It is necessary to say that a translator as an expert in Equimediation paradigm should be knowledgeable and skilful in all field. This is due to the fact that saturating the needs of the target reader will be of great importance and the translator should observe the spirit and faith of the source text. And finally, it is important to say that the agreement on the suitability of translation should not result in falsifying the content of the translation in this direction. Falsifying the content of the source text causes to eliminate the rationale of the equivalents and it makes the translation be completely superficial and fake in nature.

Equimediation paradigm uses such deep-superficial equivalents to explain the root and core of the either source or target language context. New Translation Studies seeks to conglomerate the two poles in translation. Shaded area in translation consists of some rudimental and fundamental aspects of translation. Translation is like crossing the road. It means that the translator should be in between two lines of water so as to pass the river. In translation, the renderer should consider the nature of the source text in order to clarify the nature of the target language. The translator acts like the catalyzer who seeks to connect the source to the target language context. Connecting the two bridges should be carried out cautiously because the risk of falsifying the source text is at high stake. Unlike passing the river, the translator should hybridize the source to the target languages to find out the common points in these poles. Therefore, inclination to the source and target languages should be performed equally at the same time. Whether the translator inclines more to either source-target or deep-surface, she/he will walk on the rim of falsification issue in translation.

Equivalents in Equimediation paradigm are in contact with one another. For example, in description paradigm, the translator encounters the various equivalents in the target language but she/he does not know the situation of the 
intended equivalents. Multiplicity of equivalents is not the sole factor in translation of the source language. All proposed equivalents should be context-based. In Equimediation paradigm, the relevant multitude of equivalents in gray areas is proposed in order to make translation in accordance with the target regulations. In these kinds of situations, brittle equivalents will be suggestible in essence. This is due to the fact that some equivalents carry the essence of the source in the target language and some others are target-based in nature. Keeping these sorts of equivalents leads to producing high quality translation at final stage.

One example to reveal the real function of Equimediation paradigm is Godard's (1995) experience. Goddard (1995) experienced translating one title of novel in the target language. He utilizes one unbelievable technique to transfer the main essence of the source language text. His technique is in accordance with the Equimediation paradigm. However, most of the translators are not aware of the fact that translation involves some various equivalents which are completely applicable and feasible for the target reader. It is better to note that translation of titles differs from the text per se. Every title covers some aspects of equivalence paradigm including more than one equivalent in the target language. Goddard translated a French novel into English. The name of the novel was "L'amer". During the translation of the intended novel, the translator pay attention to the fact that "L'amer" could be rendered by more than one equivalent in the target language. Goddard seeks to establish an agreement amongst the source, target languages, client, and the translator. Therefore, he infers that the title includes other equivalents such as "mere (mother)", "mer (sea)", and the "amer (bitter)". In order to show the feasibility of the translation of the title into the target language, he utilizes the schematic figure to transfer the translation impossibility. This schematic figure is as follows:

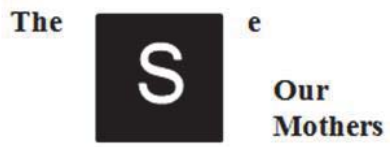

As inferred, this scheme contains some source-target equivalents namely: (1) These Our Mothers, (2) The Sour Mothers, and (3) The Sea Smothers. The term Mother and Mere share some common parts in translation. They emanate from the same source. They are categorized in partial wafting in New Translation Studies. Akbari (2014a) defines wafting as "a direct and intense translation process, it has a connection with rendering or transferring as the original SL by inserting or absorbing the SL words or phrases form into the RL's in accordance with prestigious, frequency, and intensity of the intended words (p. 27)." In this example, 'mere' and 'mother' are treated partially and they carry the same sense in source-target translation. This technique puts translation in between. Translator can exploit source-target amalgamation terms in his/her translation.

It is important to say that in the circle of translation, translator and the client are the two decision makers respectively. New Translation Studies let the reader decide the feasibility and practicability of the target text. As another example, "ogni paese sa di diritto umano" will be translated as "every country knows the huMan right". In this example, the translator utilizes the capital "M" to show that "man" in human is not pertained to the masculine sex. But, the translator prefers to address the man and woman. Therefore, he puts himself in Equimediation paradigm to show the equality of rights both deep and surface layer of the language. In this direction, positioning the reader in the circle of translation is a technique which Equimediation paradigm tries to achieve.

Currently, good translation utilizes the technique of ambiguity in translation. It should be noted that ambiguity is not the means of content falsification. Ambiguity alludes to the different inference but related to the text. This is because of the fact that the reader and the translator are the primary element in Equimediation paradigm. Ambiguity in a sense lets the reader infer what he/she seeks to ruminate about the text. Spirit, content, and faithfulness to the source and target language are three items which should be observed during the act of translating. Therefore, ambiguity scrutinizes these elements in translation so as to make an indelible translation. Needless to say, multiplicity of meaning of an item makes translation more meaningful. Afterward, the reader ponders about the multitudes of meaning and equivalents in the target language context and he/she can judge easily on workability of Equimediation paradigm in this direction.

There might be the question which occupies the reader's mind in which why the surface layer of Equimediation paradigm is wavy and the deep layer is linear in essence. It should be noted that every translation has some void and shaded areas which plays the role of translation decoration. For instance, cliché metaphors, slangs, colloquial conversations, and so forth are temporal in nature and the speaker uses them for a short period of time. They never last long in translation or daily conversation. Therefore, they might be used for translation decoration as well. That is because 
of this reason that the surface layer is wavy. Everything in the surface layer is exposed to change to beautify the target text. In this direction, weak equivalents and brittle equivalents along with low intensity of equivalents are used as the surface layer of the target text. This does not mean to say that weak and brittle equivalents are not useful or drastic in translation. But, it should be said that weak and brittle will be replaced by the other weak and brittle equivalents in a short period of time. It is like the circle which the old equivalents are replaced by the new ones. On the other hand, the deep layer is linear in nature. One has to say that strong equivalents are not exposed to change. Some items in either source or target language context are never changed during the time. For instance, beliefs, creeds, nobility, and dignity play the key roles in translation and the translator should observe their way of translating. Overall speaking, the translator has to deal with the deep structure and then deals with the surface layer of the target text. An example is given to clarify the nature of the surface and deep layer of the source and target language contexts. This is the poem rendered by the Persian translator. The former observes the deep-surface amalgamation and the latter utilized literal meaning of the source text. The English poem is as follow:

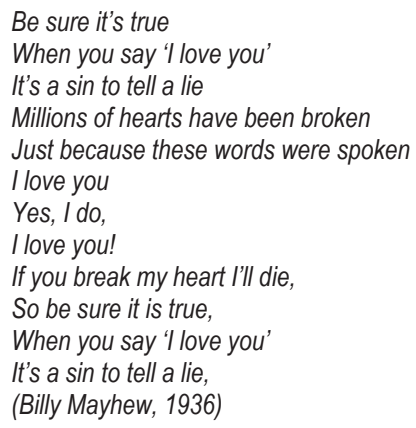

The intended Persian rendering by Sohofi (2014) with observance both deep-surface and source-target amalgamation is as follows:

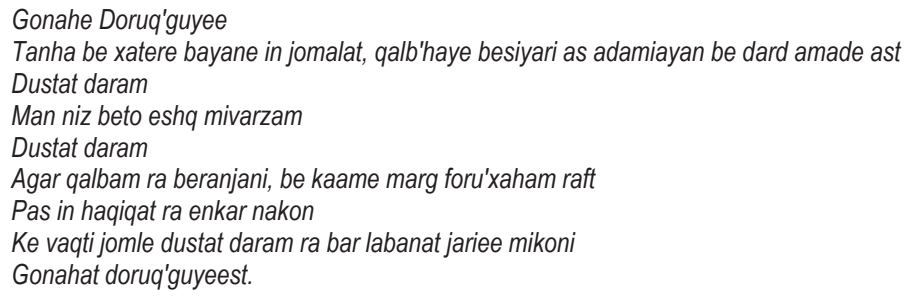

The intended translation first considers the role of the source text in the target context. Second, it regards the deep layer of the content since she translates the foundation of the source text. For the decoration of the target translation, the translator utilizes the technique of amplification in that she adds some elements in order for the readers depict the whole framework of the source text. Another point is that the translator uses fewer sentences than the original version of the poem. The original poem includes twelve sentences to convey the sense of the source text. On the other hand, the Persian rendering is translated by ten sentences and it convinces the target reader by its clarity, brevity, and feasibility. It can be stated that the ultimate aim of Equimediation paradigm is to produce high quality of translation. High quality of translation means that the translator covers the source, target languages and intention of the reader and context as the core elements in the translation.

Las but not least, Equimediation paradigm in translation treats source and target language at the same time. It is thoroughly a synchronous activity which inspects the characteristics of both source and target with each other. The translator is always a mediator since he/she is not allowed to incline more to one side. The translator always walks exactly between the two poles. Source-Target priority is always utilized so that the translator is able to create high quality of translation. It is better to recommend that Equimediation paradigm is quietly applicable in the new terms or information in translation. For instance, some new words in English might be rendered correctly via the process of Equimediation paradigm. New information might be new terms in the other language which enter English culture and communication. The translator is also free to opt for wafting procedure as the sole base in Equimediation paradigm. Wafting procedure 
inspects both the deep and surface layer of the target equivalents. The terms such as soap opera, Seifenoper, Gegenschein, Ersatz, and Levi's ${ }^{\circledR}$ are the examples of the Source-Target priority.

Equimediation paradigm in translation might be feasible in Machine Translation since it scrutinizes the frequency of occurrence and the degree of intensity of the words. Repetition of the intended equivalents to be strong enough in nature and most of the Machine translation Softwares use it for most part of the target translation. And the degree of intensity shows the rational and functions of the equivalent in the target language. Function should be translated prior to the act of translating. Machine Translation and the translator should first render the abstract idea of the source text and then walk on the road of visible parts in translation. Overall speaking, Equimediation paradigm in translation might be used in every field of study. Every field has its own decoding ability in which Equimediation paradigm can be a drastic tool so that the translator is able to persuade the reader on feasibility, practicability and functionality of the target text.

\section{Conclusion}

Choosing an approximate equivalent is an ordeal task for the translator in Translation Studies. Generally speaking, the translator's product is to persuade the reader about feasibility, applicability, and practicability of the target text. Therefore, the translator should consider the role of source text in his/her translation as well as target language context. However, most of the translators without considering the intention and rationale behind the intended equivalents, translate literally so that the reader encounters the superficial understanding of the source text. Intention and rationale behind the text transfer the rationale behind the words [equivalents]. So, the translator should make his/her efforts to convey the essence of the text prior to act of translating.

In the light of these explanations, this study seeks to introduce an equivalence paradigm known as Equimediation Paradigm. Equimediation paradigm inspects equivalents into two ways: (1) Deep-Surface layer of language and (2) Source-Target amalgamation language context. The intended paradigm pays special attention to the role of the client, the decoding ability, the translator, and the source-text function. It aims at clarifying the concealed rationale and intention of the source-target language text. The translator will be treated as an expert seeking to substantiate the nature of homogeneity in translation.

\section{References}

Akbari, A. R. (2013). Exploring into the New Model Procedure in Translation: Wafting as a Case in Point. International Journal of Education and Literacy Studies(1).

Akbari, A. R. (2014a). An Overall Perspective of Machine Translation with its Shortcomings. International Journal of Education and Literacy Studies,(1).

Akbari, A. R. (2014b). The Perks of Politeness Translation Studies. Theory and Practice in Language Studies, (4), (6), $1193-1200$.

Amman, M. \& Vermeer, H. (1994). Entwurf eines Curriculuns für einen Studiengang Translatologie und Translatorik. Heidelberg: Institut für Übersetzen und Dolmetschen, Universät Heidelberg.

Catford, J. C. (1965). A linguistic theory of translation. Oxford: Oxford Translation Press.

Chesterman, A. (1996). On Similarity, Target,(8),(1).

Chesterman, A. (2005). Where is Similarity? In S. Arduini and R. Hodgson (eds) Similarity and Difference in translation, Rimini: Guaraldi.

Delisle, J. (1988). Translation: An Interpretive Approach, trans, P. Logan and M. Creery, Ottawa: University Press.

Delabastita, D. (2008). Status, origin, features. Translation and beyond. In Beyond Descriptive Translation Studies: Investigations in homage to Gideon Toury, A. Pym, M.Shlesinger and D. Simeoni (eds). Amsterdam and Philadelphia: Benjamins. 233-246.

Goddard, B. (1995). A Translator's Driary, in Simon, S. (eds.) Culture in Transit: Translation and Changing Identities of Quebec Literature. Montreal: Vehicule Press.

Gouadec, D. (2007). Translation as Profession. Amsterdam: Philadelphia: Benjamins.

Holz-Mantari, J. (1984). Translatorisches Hande. In. Theorie und Methode. Helsinki: Anales Academicae Scentiarum Fennicae.

House, J. (1997). A Model for Translation Quality Assessment. Tübingen: Gunter Narr.

Jäger, G. (1989). Möglichkeiten und Grenzen des Äquivalenzbeziehungsmodells bei der Erklärung der Translation. In Linguistische Arbeitsberichte 67. Sektion Theoretische und Angewandte Sprachwissenschaft. Leipzig: Karl-Marx-Universität.

Nida, E.A. (1986). From One Language to Another: Functional Equivalence in Bible Translating. Nashville: Thomas Nelson Publishers.

Nida, E. A., \& Taber, C. R. (1969). The theory and practice of translation. Leiden: E. J. Brill.

Nord, C. (1988) Translating as a Purposeful Activity. Functionalist Approaches Explained. Manchester: St. Jerome.

Nord, C. (1997) Translating as a Purposeful Activity. Functionalist Approaches Explained, Manchester: St. Jerome.

Snell-Hornby, M; Honig, H.G; Kussmaul, P; Schmidt, P (ed) (1999). Handbuch Translation. Tübingen: Stauffenburg Verlag.

Toury, G. (1985). A Rationale for Descriptive Translation Studies. In T. Hermans(Ed.), The Manipulation of Literature (pp. 16-41). London and Sydney:Croom Helm.

Toury, G. (1995). Descriptive translation studies - and beyond, Amsterdam and Philadelphia, PA: John Benjamins. 
Vermeer, H. (1978). Skopos and commission in translational activity. In: Venuti, L. The Translation Studies Reader. Routledge: London. Vermeer, Hans J. (1989a). "Skopos and Commission in Translational Action." Heidelberg: Vermeer.

Vermeer, H.J. (1989b/2004). Skopos and Commission in Translational Action, in L. Venuti (ed.) The Translation Studies Reader, second edition, London, New York: Routledge.

Wills, W. (1982). The science of translation. Stuttgart: Gunter Narr Verlag Tubingen. 